\title{
SIMULTENIOUS APPLICATION OF THE BOUNDARY ELEMENT METHOD AND THE POWER SERIES METHOD FOR SOLVING A TWO-DIMENSIONAL PROBLEM OF HEAT WAVE MOTION
}

\author{
A. L. Kazakov ${ }^{1}$, L. F. Spevak ${ }^{2}$, O. A. Nefedova ${ }^{2} *$ \\ ${ }^{\text {I} M a t r o s o v}$ Institute for System Dynamics and Control Theory of Siberian Branch of Russian Academy of Sciences, \\ 134 Lermontov st., Irkutsk, Russian Federation \\ ${ }^{2}$ Institute of Engineering Science, Ural Branch of the Russian Academy of Sciences, \\ 34 Komsomolskaya st., Ekaterinburg, Russian Federation \\ *Corresponding author. E-mail: nefedova@imach.uran.ru \\ Address for correspondence: ul. Komsomolskaya, 34, Ekaterinburg, Russian Federation \\ Tel.: +7 (343) 37535 92; fax: +7 (343) 3745330
}

The paper develops numerical solution methods for heat conduction boundary value problems for the case of the power dependence of the heat conductivity factor on temperature. Besides heat distribution in space, it describes filtration of a polytrophic gas in a porous medium. A distinctive feature of this equation is the degeneration of its parabolic type when the required function becomes zero, whereupon the equation acquires some properties typical of first-order equations. Particularly, in some cases, it proves possible to substantiate theorems of the existence and uniqueness of heat-wave type solutions for it. A numerical method using the advantages of the power series method and the boundary element method is proposed for the solution of the boundary value problem with a specified heat wave front. Simultaneous application of the two methods allows the accuracy of the numerical solution to be increased. A program has been developed from the proposed method. Test examples are considered.

Keywords: nonlinear heat conduction equation, power series, boundary element method, computational experiment.

DOI: $10.17804 / 2410-9908.2017 .6 .006-015$

\section{References}

1. Vazquez J.L. The Porous Medium Equation: Mathematical Theory. Clarendon Press, Oxford, 2007, 648 p. ISBN-10: 0198569033, ISBN-13: 978-0198569039.

2. Samarsky A.A., Galaktionov V.A., Kurdyumov S.P. Mikhailov A.P. Rezhimy s Obostreniem $v$ Zadachakh dlya Nelineinykh Parabolicheskikh Uravneniy [Regimes with Peaking in Problems for Quasilinear Parabolic Equations]. Moscow, Nauka Publ., 1987, 476 p. (In Russian).

3. Sidorov A.F. In: Izbrannye Trudy: Matematika. Mekhanika [Selected Works: Mathematics. Mechanics]. Moscow, Fizmatlit Publ., 2001, 576 p. (In Russian). ISBN 5-9221-0103-X.

4. Kazakov A.L., Spevak L.F. Numerical and analytical studies of a nonlinear parabolic equation with boundary conditions of a special form. Applied Mathematical Modelling, 2013, vol. 37, iss. 10-11, pp. 6918-6928. DOI: 10.1016/j.apm.2013.02.026

5. Kazakov A.L., Spevak L.F. An analytical and numerical study of a nonlinear parabolic equation with degeneration for the cases of circular and spherical symmetry. Applied Mathematical Modelling, 2015, vol. 40, iss. 2, pp. 1333-1343. DOI: 10.1016/j.apm.2015.06.038

6. Spevak L.F., Nefedova O.A. Solving a two-dimensional nonlinear heat conduction equation with degeneration by the boundary element method with the application of the dual reciprocity method. In: AIP Conference Proceedings, 2016, vol. 1785, iss. 1, pp. 040077. Available at: http://doi.org/10.1063/1.4967134

7. Kazakov A.L., Spevak L.F., Nefedova O.A. Solution of a Two-Dimensional Problem on the Motion of a Heat Wave Front with the Use of Power Series and the Boundary Element Method. Izvestiya IGU. Seriya Matematika. Mekhanika, 2016, Vol. 18, pp. 21-37 (In Russian). 
8. Brebbia C.A., Telles J.F.C., Wrobel L.C. Boundary Element Techniques. SpringerVerlag, Berlin, Neidelberg, New-York, Tokyo, 1984, 466 p. ISBN: 978-3-642-48862-7 (Print), 978-3-642-48860-3 (Online). DOI: 10.1007/978-3-642-48860-3

9. Fedotov V.P., Spevak L.F. One approach to the derivation of exact integration formulae in the boundary element method. Engineering Analysis with Boundary Elements, 2008, vol. 32, no. 10, pp. 883-888. DOI: $10.1016 /$ j.enganabound.2008.03.001

10. Nardini D., Brebbia C.A. A New Approach to Free Vibration Analysis using Boundary Elements. Applied Mathematical Modelling, 1983, vol. 7, no. 3, pp. 157-162. DOI: 10.1016/0307-904X(83)90003-3 
Подана в журнал: 16.11 .2017

УДК 517.958:519.633

DOI: $10.17804 / 2410-9908.2017 .6 .006-015$

\title{
СОВМЕСТНОЕ ИСПОЛЬЗОВАНИЕ МЕТОДА СТЕПЕННЫХ РЯДОВ И МЕТОДА ГРАНИЧНЫХ ЭЛЕМЕНТОВ ДЛЯ РЕШЕНИЯ ДВУМЕРНОЙ ЗАДАЧИ О ДВИЖЕНИИ ФРОНТА ТЕПЛОВОЙ ВОЛНЫ
}

\author{
А. Л. Казаков ${ }^{1}$, Л. Ф. Спевак ${ }^{2}$, О. А. Нефедова ${ }^{2} *$ \\ ${ }^{1}$ Федеральное государственное бюджетное учреждение науки Институт динамики систем и теории \\ управления имени В.М. Матросова Сибирского отделения Российской академии наук, \\ 664033, ул. Лермонтова, 134, Иркутск, Российская Федерация \\ ${ }^{2}$ Федеральное государственное бюджетное учреждение науки Институт машиноведения \\ Уральского отделения Российской академии наук, \\ 620049, ул. Комсомольская, 34, Екатеринбург, Российская Федерация \\ *Ответственный автор. Электронная почта: nefedova@imach.uran.ru \\ Адрес для переписки: 620049, ул. Комсомольская, 34, Екатеринбург, Российская Федерация \\ Тел.: +7 (343) 375-35-92; факс: +7 (343) 374-53-30
}

Работа посвящена разработке численных методов решения краевых задач для нелинейного уравнения теплопроводности в случае степенной зависимости коэффициента теплопроводности от температуры. Помимо распространения тепла в пространстве, это уравнение описывает также фильтрацию политропного газа в пористой среде. Особенностью рассматриваемого уравнения является вырождение его параболического типа в случае нулевого значения искомой функции, вследствие чего уравнение приобретает некоторые свойства, обычно характерные для уравнений первого порядка. В частности, для него в некоторых случаях удается обосновать теоремы существования и единственности решений типа тепловой волны. Для краевой задачи с заданным фронтом тепловой волны предложен метод решения, использующий преимущества ранее применявшихся метода степенных рядов и метода граничных элементов. Совместное применение двух методов позволило повысить точность численного решения. Предложенный метод реализован в виде программы для ЭВМ. Рассмотрены тестовые примеры.

Ключевые слова: нелинейное уравнение теплопроводности, степенной ряд, метод граничных элементов, вычислительный эксперимент.

\section{1. Введение}

Разработка новых численных методов решения нелинейных краевых задач для уравнений математической физики, описывающих распространение тепла в пространстве, остается актуальной проблемой прикладной математики. Рассмотрим нелинейное уравнение теплопроводности

$$
T_{t}=\alpha \operatorname{div}(k(T) \nabla T) .
$$

Здесь $t$ - время, $T$ - искомая функция (температура); $\alpha$ - положительные константы, $k(T)$ - коэффициент теплопроводности; $\operatorname{div}, \nabla$ - дивергенция и градиент по пространственным координатам. В случае степенной зависимости коэффициента теплопроводности от температуры $[1,2]$

$$
k(T)=T^{\sigma}, \sigma>0
$$


уравнение (1.1) стандартной подстановкой $u=T^{\sigma}, t^{\prime}=\alpha t$ сводится к уравнению

$$
u_{t}=u \Delta u+\frac{1}{\sigma}(\nabla u)^{2}
$$

Одномерные краевые задачи для уравнения (1.3) рассматривались, в частности, в работе [3]. В предыдущих работах $[4,5]$ для случаев плоской, круговой и сферической симметрии при различных краевых режимах авторами были доказаны теоремы существования и единственности аналитического решения в виде степенного ряда. Также в этих работах были построены алгоритмы численного решения задач на заданном конечном промежутке времени с помощью метода граничных элементов (МГЭ). Применение метода степенных рядов для конструктивного доказательства теоремы существования и единственности решения и алгоритмы решения МГЭ для случая двух пространственных переменных представлены в работах $[6,7]$. Хорошо известно, что аналитические методы решения нелинейных задач математической физики, к которым относится метод степенных рядов, обладают весьма ограниченными возможностями для использования. Все теоремы существования и единственности, которые упоминались выше, являются локальными, а аналитические решения в виде степенных рядов являются решениями в окрестности начального момента времени. Оценка радиуса сходимости этих рядов требует отдельного исследования в каждой конкретной задаче. В свою очередь построенные в работах [4-7] алгоритмы численного решения на конечном промежутке времени с помощью МГЭ показали свою эффективность. Однако на первом шаге по времени существуют определенные трудности при разностном вычислении производной искомой функции по времени.

В работе предлагается, используя преимущества обоих подходов, на первом шаге по времени использовать аналитическое решение, что позволит получить численное решение на следующих шагах с более высокой точностью.

\section{2. Постановка краевой задачи}

Уравнение (1.3) в двумерном случае имеет вид:

$$
u_{t}=u\left(u_{x_{1} x_{1}}+u_{x_{2} x_{2}}\right)+\frac{1}{\sigma}\left(u_{x_{1}}^{2}+u_{x_{2}}^{2}\right) .
$$

Зададим краевое условие в виде

$$
\left.u\right|_{b\left(t, x_{1}, x_{2}\right)=0}=0,
$$

где уравнение $b\left(t, x_{1}, x_{2}\right)=0$ в каждый момент времени определяет нулевой фронт тепловой волны $S^{(t)}$ - замкнутую гладкую линию, ограничивающую область $V^{(t)}$, содержащую начало координат. Предполагается, что если $t_{1}<t_{2}$, то $V^{\left(t_{1}\right)} \subset V^{\left(t_{2}\right)}$. Задача состоит в определении функции $u=u\left(t, x_{1}, x_{2}\right)$ в области $t \in\left[0, t_{*}\right], x\left(x_{1}, x_{2}\right) \in \Omega^{(t)}$, где $\Omega^{(t)}$ - область, ограниченная границами $S^{(0)}$ и $S^{(t)}$.

Можно показать, что при выполнении условия (2.2) справедливо соотношение

$$
\left.q\right|_{b\left(t, x_{1}, x_{2}\right)=0}=\frac{\sigma b_{t}\left(t, x_{1}, x_{2}\right)}{\sqrt{b_{x_{1}}^{2}\left(t, x_{1}, x_{2}\right)+b_{x_{2}}^{2}\left(t, x_{1}, x_{2}\right)}},
$$


где $q=\frac{\partial u}{\partial n}$ - тепловой поток, $n$ - вектор внешней нормали к границе рассматриваемой области в момент времени $t$.

\section{3. Аналитическое решение методом степенных рядов}

Для построения аналитического решения методом степенных рядов запишем уравнение (2.1) и краевое условие (2.2) в полярных координатах $\rho, \varphi$ :

$$
\begin{gathered}
u_{t}=u u_{\rho \rho}+\frac{1}{\sigma} u_{\rho}^{2}+\frac{1}{\rho} u u_{\rho}+\frac{1}{\rho^{2}}\left(\frac{u_{\varphi}^{2}}{\sigma}+u u_{\varphi \varphi}\right), \\
\left.u\right|_{\rho=b(t, \varphi)}=0 .
\end{gathered}
$$

Здесь $x_{1}=\rho \cos \varphi, x_{2}=\rho \sin \varphi$. Для упрощения обозначений для функции, задающей движение фронта тепловой волны, сохранено прежнее обозначение $b$. Для задачи (3.1) и (3.2) справедлива следующая теорема [7].

Теорема 1. Пусть в задаче (3.1), (3.2) функиия $b(t, \varphi)$ обладает свойствами:

1. $b(t,-\pi)=b(t, \pi), b(t, \varphi)>0$.

2. $b(t, \varphi)$ и $1 / b(t, \varphi)$ являются аналитическими по $\varphi$ при $-\pi \leq \varphi \leq \pi$ и по $t$ в некоторой окрестности начального момента времени $t=0$.

Тогда задача (3.1) и (3.2) в некоторой окрестности замкнутой линии $\rho=b(0, \varphi),-\pi \leq \varphi \leq \pi$ имеет единственное локальное по $t$ нетривиальное аналитическое решение.

Доказательство теоремы проведено конструктивно, построением аналитического решения в виде степенного ряда

$$
u=\sum_{k=0}^{\infty} \frac{u_{k}(t, \varphi)}{k !} s^{k}
$$

где $s=\rho-b(t, \varphi) ; u_{k}=\left.\frac{\partial^{k} u}{\partial s^{k}}\right|_{s=0}$. Коэффициенты $u_{k}$ определяются рекуррентно [7].

\section{4. Алгоритм решения МГЭ}

Численное решение задачи (2.1) и (2.2) производится по шагам по времени. На каждом текущем шаге, соответствующем моменту времени $t$, в области $\Omega^{(t)}$ рассматривается уравнение Пуассона

$$
u_{x_{1} x_{1}}+u_{x_{2} x_{2}}=\frac{1}{u}\left(u_{t}-\frac{u_{x_{1}}^{2}+u_{x_{2}}^{2}}{\sigma}\right)
$$

с граничными условиями, вытекающими из условий (2.2), (2.3):

Kazakov A.L. et al. / Simultenious application of the boundary element method and the power series method for solving a two-dimensional problem of heat wave motion 


$$
\begin{gathered}
\left.u\right|_{x \in S^{(t)}}=0 ; \\
\left.q\right|_{x \in S^{(t)}}=\frac{\sigma b_{t}}{\sqrt{b_{x_{1}}^{2}+b_{x_{2}}^{2}}} .
\end{gathered}
$$

Решение задачи (4.1)-(4.3) методом граничных элементов приводит к соотношению

$$
u(\xi)=\int_{S}\left[q(x) u^{*}(\xi, x)-u(x) q^{*}(\xi, x)\right] d S(x)-\int_{\Omega^{(t)}} \frac{1}{u}\left(u_{t}-\frac{u_{x_{1}}^{2}+u_{x_{2}}^{2}}{\sigma}\right) u^{*}(\xi, x) d \Omega(x),
$$

где $\xi$ - внутренняя точка области $\Omega^{(t)}, S=S^{(0)} \cup S^{(t)}, u^{*}(\xi, x)$ - фундаментальное решение, $q^{*}(\xi, x)=\frac{\partial u^{*}(\xi, x)}{\partial n}[8]$. Для граничной точки $x_{0} \in S$ справедливо

$$
\frac{1}{2} u\left(x_{0}\right)=\int_{S}\left[q(x) u^{*}\left(x_{0}, x\right)-u(x) q^{*}\left(x_{0}, x\right)\right] d S(x)-\int_{\Omega} \frac{1}{u}\left(u_{t}-\frac{u_{x_{1}}^{2}+u_{x_{2}}^{2}}{\sigma}\right) u^{*}\left(x_{0}, x\right) d \Omega(x) .
$$

Разбивая границу $S$ на $N$ граничных элементов и записывая уравнение (4.5) для каждого узла $\xi_{i}$, получаем систему линейных уравнений для определения узловых значений температуры и потока, не заданных граничными условиями (4.2) и (4.3):

$$
\begin{gathered}
\frac{1}{2} u_{i}=\sum_{j=1}^{N}\left(q_{j} \int_{e_{j}} u^{*}\left(\xi_{i}, x\right) d x-u_{j} \int_{e_{j}} q^{*}\left(\xi_{i}, x\right) d x\right)-\int_{\Omega^{(t)}} \frac{1}{u}\left(u_{t}-\frac{u_{x_{1}}^{2}+u_{x_{2}}^{2}}{\sigma}\right) u^{*}\left(\xi_{i}, x\right) d \Omega(x), \\
i=1, \ldots, N
\end{gathered}
$$

Здесь $u_{i}, q_{i}$ - значения температуры и потока в узле $\xi_{i}$ элемента $e_{i}, i=1, \ldots, N$. На каждом элементе принята постоянная аппроксимация искомой функции и потока. Интегралы по граничным элементам вычисляются точно, по полученным в [9] аналитическим формулам.

Для вычисления интегралов, стоящих в правых частях уравнений (4.4)-(4.6), применялся метод двойственной взаимности [10]. Входящий в подынтегральные выражения множитель $\frac{1}{u}\left(u_{t}-\frac{u_{x_{1}}^{2}+u_{x_{2}}^{2}}{\sigma}\right)$ представлялся в виде

$$
\frac{1}{u}\left(u_{t}-\frac{u_{x_{1}}^{2}+u_{x_{2}}^{2}}{\sigma}\right)=\sum_{i=1}^{M} \alpha_{i} f_{i}(x),
$$

где для функций $f_{i}$ существуют такие функции $\hat{u}_{i}$, что $f_{i}=\Delta \hat{u}_{i}$. В качестве функций $f_{i}$ применялись радиальные базисные функции, значения которых зависят от расстояния между текущей точкой и заданными точками коллокации $y_{1}, y_{2}, \ldots, y_{M}$, лежащими в области $\Omega^{(t)}$ : $f_{i}(x)=f_{i}\left(r_{i}\right)$, где $r_{i}=\left\|x-y_{i}\right\|$.

Kazakov A.L. et al. / Simultenious application of the boundary element method and the power series method for solving a two-dimensional problem of heat wave motion 
С учетом (4.7), интегралы могут быть вычислены следующим образом:

$$
\begin{gathered}
\int_{\Omega} \frac{1}{u}\left(u_{t}-\frac{u_{x_{1}}^{2}+u_{x_{2}}^{2}}{\sigma}\right) u^{*}(\xi, x) d \Omega(x)=\sum_{i=1}^{M} \alpha_{i} \int_{\Omega} f_{i}(x) u^{*}(\xi, x) d \Omega(x)= \\
=\sum_{i=1}^{M} \alpha_{i} \int_{\Omega} \Delta a_{i}(x) u^{*}(\xi, x) d \Omega(x)=\sum_{i=1}^{M} \alpha_{i}\left(-a_{i}(\xi)+\int_{S}\left[\hat{a}_{i}(x) u^{*}(\xi, x)-a_{i}(x) q^{*}(\xi, x)\right] d S(x)\right),
\end{gathered}
$$

где $\hat{q}_{i}(x)=\frac{\partial \hat{u}_{i}(x)}{\partial n}$. Все вычисления, таким образом, сводятся на границу области решения задачи, и основное преимущество МГЭ - уменьшение размерности задачи - сохраняется. Полученные соотношения (4.4)-(4.8) позволяют решить исходную задачу (2.1) и (2.2) по шагам по времени. На каждом шаге задача (4.1)-(4.3) итерационно решается следующим образом. В качестве начального приближения принимается тривиальное решение. Для найденной $n$-й итерации решения $u^{(n)}(x)$ вычисляются значения левой части соотношения (4.7) в точках коллокации, составляется система линейных алгебраических уравнений относительно коэффициентов $\alpha_{i}$ :

$$
\left.\frac{1}{u^{(n)}}\left(u_{t}^{(n)}-\frac{\left(u_{x_{1}}^{(n)}\right)^{2}+\left(u_{x_{2}}^{(n)}\right)^{2}}{\sigma}\right)\right|_{x=y_{k}}=\sum_{i=1}^{M} \alpha_{i} f_{i}\left(y_{k}\right), k=1, \ldots, M
$$

Решив систему (4.9), находим соответствующие текущей итерации коэффициенты $\alpha_{1}^{(n)}, \alpha_{2}^{(n)}, \ldots, \alpha_{M}^{(n)}$. Далее решается система граничных интегральных уравнений:

$$
\begin{gathered}
\frac{1}{2} u_{i}=\sum_{j=1}^{N}\left(q_{j} \int_{e_{j}} u^{*}\left(\xi_{i}, x\right) d x-u_{j} \int_{e_{j}} q^{*}\left(\xi_{i}, x\right) d x\right)- \\
-\sum_{m=1}^{M} \alpha_{m}^{(n)}\left(-a_{m}\left(\xi_{i}\right)+\sum_{j=1}^{N}\left(\hat{q}_{m}\left(\xi_{i}\right) \int_{e_{j}} u^{*}\left(\xi_{i}, x\right) d x-a_{m}\left(\xi_{i}\right) \int_{e_{j}} q^{*}\left(\xi_{i}, x\right) d x\right)\right), i=1, \ldots, N .
\end{gathered}
$$

Решение последней системы, узловые значения $u_{i}^{(n+1)} ; q_{i}^{(n+1)}$, определяют следующую $(n+1)$-ю итерацию:

$$
\begin{gathered}
u^{(n+1)}(\xi)=\sum_{j=1}^{N}\left(q_{j}^{(n+1)} \int_{e_{j}} u^{*}\left(\xi_{i}, x\right) d x-u_{j}^{(n+1)} \int_{e_{j}} q^{*}\left(\xi_{i}, x\right) d x\right)- \\
-\sum_{m=1}^{M} \alpha_{m}^{(n)}\left(-\hat{u}_{m}\left(\xi_{i}\right)+\sum_{j=1}^{N}\left(\hat{q}_{m}\left(\xi_{i}\right) \int_{e_{j}} u^{*}\left(\xi_{i}, x\right) d x-\hat{u}_{m}\left(\xi_{i}\right) \int_{e_{j}} q^{*}\left(\xi_{i}, x\right) d x\right)\right), i=1, \ldots, N .
\end{gathered}
$$
итераций.

Итерационный процесс заканчивается при достаточной близости двух последовательных

Kazakov A.L. et al. / Simultenious application of the boundary element method and the power series method for solving a two-dimensional problem of heat wave motion 
Наибоее тонким моментом представленного алгоритма с вычислительной точки зрения является нахождение производной по времени $u_{t}$ в точках коллокации в уравнениях (4.9). Она вычисляется разностно, с использованием решения на предыдущем шаге по времени. При этом на первом шаге приходится отталкиваться от нулевого значения, что может привести к дополнительной погрешности вычислений. В связи с этим в данной работе предлагается проводить численное решение по представленному алгоритму начиная со второго шага по времени, а на первом шаге, находящемся вблизи начального момента времени, находить производную по времени дифференцированием локального аналитического решения в виде отрезка ряда (3.3). Таким образом мы можем использовать преимущества двух методов.

\section{5. Примеры}

Верификация предложенного алгоритма проводилась сравнением численных решений с известным точным решением в симметричном случае, когда нулевой фронт является окружностью:

$$
b\left(t, x_{1}, x_{2}\right)=x_{1}^{2}+x_{2}^{2}-(R(t))^{2} .
$$

В случае, когда радиус фронта изменяется в соответствии с зависимостью

$$
R(t)=R\left(1+\frac{\mu t}{C}\right)^{v},
$$

задача (2.1) и (2.2) имеет точное решение:

$$
u=-\frac{x_{1}^{2}+x_{2}^{2}}{C+\mu t}+\frac{C^{k-1} R^{2}}{(C+\mu t)^{k}} .
$$

Здесь $\mu=4+\frac{4}{\sigma} ; v=\frac{1}{2 \sigma+2} ; k=\frac{4}{\mu} ; R$ и $C$ - заданные положительные константы.

Численное решение представленной тестовой задачи, предложенным в данной работе методом сравнивалось с решением методом граничных элементов $[6,7]$. Были приняты следующие значения параметров: $\sigma=3 ; R=3 ; C=10$. В таблице приведены относительные погрешности решений с шагами по времени $h=0,05$ и $h=0,1$ на внутренней границе $S^{(0)}: x_{1}^{2}+x_{2}^{2}=R^{2}$ в различные моменты времени. Полученные результаты показывают, что совместное использование методов степенных рядов и граничных элементов вблизи начального момента времени позволило повысить точность расчетов.

Таблица. Относительные погрешности численных решений

\begin{tabular}{|c|c|c|c|c|}
\hline \multirow{2}{*}{$\begin{array}{c}\text { Момент } \\
\text { времени }\end{array}$} & \multicolumn{2}{|c|}{$\begin{array}{c}\text { Решение методом граничных } \\
\text { элементов }\end{array}$} & $\begin{array}{c}\text { Совместное применение мето- } \\
\text { дов степенных рядов и гра- } \\
\text { ничных элементов }\end{array}$ \\
\cline { 2 - 5 } & $h=0,05$ & $h=0,1$ & $h=0,05$ & $h=0,1$ \\
\hline$t=0,3$ & 0,003307 & 0,034676 & 0,000034 & 0,022401 \\
\hline$t=0,5$ & 0,002358 & 0,010311 & 0,000855 & 0,005826 \\
\hline$t=0,8$ & 0,003737 & 0,005921 & 0,002611 & 0,004846 \\
\hline$t=1,0$ & 0,007191 & 0,02319 & 0,002417 & 0,01591 \\
\hline
\end{tabular}

Kazakov A.L. et al. / Simultenious application of the boundary element method and the power series method for solving a two-dimensional problem of heat wave motion 
Также была решена задача для эллиптического нулевого фронта

$$
b\left(t, x_{1}, x_{2}\right)=\frac{x_{1}^{2}}{a_{1}(t)^{2}}+\frac{x_{2}^{2}}{a_{2}(t)^{2}}-1
$$

при линейном зависимости полуосей эллипса от времени: $a_{1}(t)=c a_{2}(t)=c(1+\alpha t)$. На рисунке показаны результаты расчетов при $\sigma=3 ; c=1,5 ; \alpha=0,05$. Решение задачи проводилось с шагом по времени $h=0,1$.

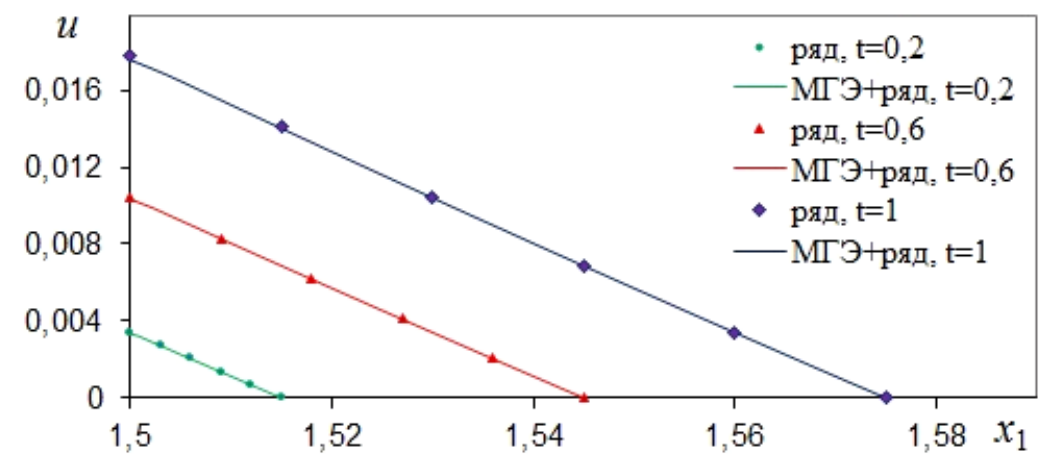

$a$

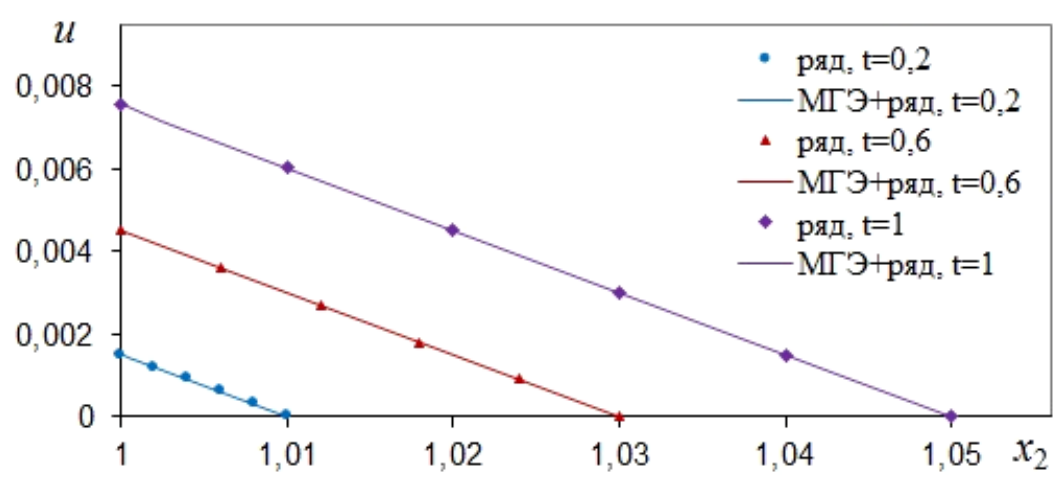

$\sigma$

Сравнение решения методом степенных рядов и решения при совместном применении методов степенных рядов и граничных элементов: $a$ - вдоль оси $O x_{1} ; \sigma$ - вдоль оси $O x_{2}$.

\section{6. Заключение}

В работе предложен метод численного решения нелинейного уравнения теплопроводности при заданном нулевом фронте тепловой волны на основе совместного применения метода граничных элементов и метода степенных рядов. Использование вблизи начального момента времени аналитического решения позволило повысить точность численного решения методом граничных элементов. Проведены численные эксперименты, показавшие преимущество предложенного подхода над использованным ранее.

\section{Благодарность}

Работа выполнена при частичной поддержке Комплексной программы УрО РАН, проект № 15-7-1-17, и РФФИ, проект № 16-01-00608. 


\section{Литература}

1. Vazquez J. L. The Porous Medium Equation: Mathematical Theory. - Oxford : Clarendon Press, 2007. - 648 p. - ISBN-10: 0198569033, ISBN-13: 978-019856903.

2. Режимы с обострением в задачах для нелинейных параболических уравнений / А. А. Самарский, В. А. Галактионов, С. П. Курдюмов, А. П. Михайлов. - М. : Наука, 1987. $476 \mathrm{c}$.

3. Сидоров А. Ф. Избранные труды: Математика. Механика. - М. : Физматлит, 2001. 576 c. - ISBN 5-9221-0103-X.

4. Kazakov A. L., Spevak L. F. Numerical and analytical studies of a nonlinear parabolic equation with boundary conditions of a special form // Applied Mathematical Modelling. - 2013. Vol. 37, iss. 10-11. - P. 6918-6928. - DOI: 10.1016/j.apm.2013.02.026

5. Kazakov A. L., Spevak L. F. An analytical and numerical study of a nonlinear parabolic equation with degeneration for the cases of circular and spherical symmetry // Applied Mathematical Modelling. 2015. - Vol. 40, iss. 2. - P. 1333-1343. - DOI: 10.1016/j.apm.2015.06.038

6. Spevak L. F., Nefedova O. A. Solving a two-dimensional nonlinear heat conduction equation with degeneration by the boundary element method with the application of the dual reciprocity method // AIP Conference Proceedings. - 2016. - Vol. 1785, iss. 1. - P. 040077. URL: http://doi.org/10.1063/1.4967134

7. Казаков А. Л., Спевак Л. Ф., Нефедова О. А. Решение двумерной задачи о движении фронта тепловой волны с использование степенных рядов и метода граничных элементов // Известия ИГУ. Серия «Математика». - 2016. - Т. 18. - С. 21-37.

8. Brebbia C. A., Telles J. F. C., Wrobel L. C. Boundary Element Techniques. - Berlin, Neidelberg, New-York, Tokyo : Springer-Verlag, 1984. - 466 p. - ISBN 978-3-642-48862-7. - DOI: 10.1007/978-3-642-48860-3

9. Fedotov V. P., Spevak L. F. One approach to the derivation of exact integration formulae in the boundary element method // Engineering Analysis with Boundary Elements. - 2008. - Vol. 32, no. 10. - P. 883-888. - DOI: $10.1016 /$ j.enganabound.2008.03.001

10. Nardini D., Brebbia C. A. A New Approach to Free Vibration Analysis using Boundary Elements // Applied Mathematical Modelling. - 1983. - Vol. 7, no. 3. - P. 157-162. DOI: $10.1016 / 0307-904 X(83) 90003-3$ 